\title{
Retroperitoneal Tuberculosis in Pregnancy: A Case Report and Literature Review
}

\author{
Lara María Mateo-Mosquera, Lucía Martínez-Braña, María Carmen Beceiro-Abad, \\ Roberto García-Figueiras, Arturo González-Quintela, and José Antonio Díaz-Peromingo
}

\section{ABSTRACT}

Tuberculosis is and infectious disease that can affect multiple organs and systems. It can involve the almost the whole body including the abdomen. Retroperitoneal involvement is uncommon, especially during pregnancy. We report the case of a pregnant woman with a retroperitoneal mass whose final diagnosis was tuberculosis, focusing on the timing and difficulties to ensure the diagnosis.

Keywords: tuberculosis, retroperitoneum, pregnancy.

Published Online: November 19, 2020

ISSN: 2593-8339

DOI: $10.24018 /$ ejmed.2020.2.6.

Lara María Mateo-Mosquera

Department of Internal Medicine, Complejo Hospitalario Universitario de Santiago, Spain.

Lucía Martínez-Braña

Department of Internal Medicine, Complejo Hospitalario Universitario de Santiago, Spain.

María Carmen Beceiro-Abad,

Department of Internal Medicine, Complejo Hospitalario Universitario de Santiago, Spain.

Roberto García-Figueiras

Department of Radiology, Complejo

Hospitalario Universitario de Santiago, Spain.

Arturo González-Quintela

Department of Internal Medicine, Complejo Hospitalario Universitario de Santiago, Spain.

José Antonio Díaz-Peromingo*

Department of Internal Medicine, Complejo Hospitalario Universitario de Santiago, Spain.

(e-mail:

jose.antonio.diaz.peromingo@sergas.es)

*Corresponding Author

\section{INTRODUCTION}

Retroperitoneal masses are a group of heterogeneous lesions originating or occupying the retroperitoneal spaces. Most of cases are due to malignant tumors, most frequently from mesenchymal, neural, germ-cell, and lymphoproliferative origin. There are also non-malignant processes such as primarily fibrosis, non-Langerhans histiocytosis, extramedullary hematopoiesis, and retroperitoneal abscesses [1]. These abscesses are commonly microbial and can be secondary to a perforated colonic carcinoma, non-malignant diseases such as Crohn's disease, diverticulitis or even appendicitis, or abdominal injury. There are also other infections such as pyelonephritis, renal carbuncle or tuberculosis that can produce retroperitoneal abscesses. Abdominal tuberculosis affects commonly the intestine, the peritoneum, or lymphadenopathy [2]. We report the case of retroperitoneal mass in a pregnant woman where tubercular etiology was established after histopathological exam and microbiological culture focusing on clinical presentation and management.

\section{CASE REPORT}

A 41-year old previously healthy pregnant woman (29 weeks) was referred to the Internal Medicine outpatient clinic, because of an abdominal mass found by chance in a routine obstetric ultrasonographic study (US). The patient was asymptomatic and general examination was normal. Laboratory results revealed an elevated erythrocyte sedimentation rate (ESR; $120 \mathrm{~mm} / \mathrm{hr}$, normal range 0$29 \mathrm{~mm} / \mathrm{hr}$ ) and C-reactive protein (CRP; $80 \mathrm{mg} / \mathrm{L}$, normal range $<10 \mathrm{mg} / \mathrm{L})$ with increased leucocyte count $\left(19.000 / \mathrm{mm}^{3}\right.$, normal range $4.000-10.000 / \mathrm{mm}^{3}, 83 \%$ neutrophils, and 7\% lymphocytes), anemia (hemoglobin $11.2 \mathrm{~g} / \mathrm{L}$, normal range 12.1-15.1 g/L), and thrombocytosis 
(773.000 / $\mu \mathrm{L}$, normal range 150.000-400.000/ $/ \mathrm{L})$. Other biochemical test such as liver function test, urea nitrogen, and serum creatine were within normal limits. A chest X ray was reported as normal. Pelvic ultrasonographic study showed a 90x42x48 mm abdominal mass at left prevertebral and retroperitoneal location. Magnetic resonance confirmed the presence of a left infrarenal paraaortic mass (90x66 mm). It showed extensive cystic areas and solid portions combined with calcifications, suggesting the presence of a probable neurogenic tumor.

Pregnancy continued without problems. Prenatal ultrasonography showed the presence of a single fetus consistent with $28 \pm 2$ weeks of pregnancy. There were no changes in the size and characteristics of the maternal mass. The patient gave birth by vaginal delivery. After delivery, a CT scan was performed (Fig. 1 and 2). Exploratory laparotomy was performed. When dissecting the retroperitoneal mass, a purulent material, remembering the appearance of the caseum spilled out.

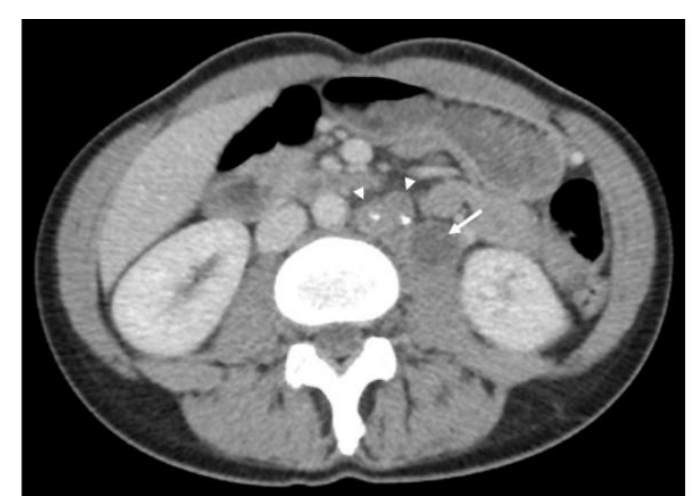

Fig. 1. The lesion shows a heterogeneous appearance with several foci of calcification inside (arrowheads) and a mass, larger, with tapered morphology and lower density (white arrow) in the transversal plane.

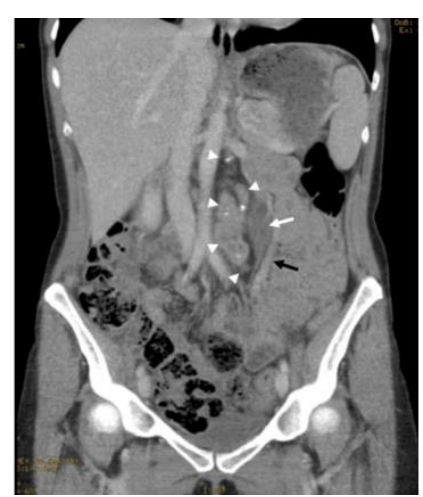

Fig. 2. Multidetector CT image reconstruction in the coronal plane shows the presence of a left para-aortic retroperitoneal mass displacing ipsilateral gonadal vein (black arrow). The lesion shows a heterogeneous appearance with several foci of calcification inside (arrowheads) and a mass, larger, with tapered morphology and lower density (white arrow).

Anatomopathological examination was consistent with the presence of granulomas with caseous necrosis. The Ziehl-Nielsen stain was negative of acid fast bacilli. Tuberculous polymerase chain reaction analysis was also negative in the biopsy specimen, but microbiological culture was positive for Mycobacterium tuberculosis. Serum HIV test was negative.

According to these findings, the patient received antitubercular chemotherapy regimen with isoniazid, rifampicin and pyrazinamide for 2 months. This treatment was followed by isoniazid and rifampicin for other four months without reporting any side effects.

Her daughter was studied without any signs of tuberculous infection at birth and during the next 6 months of follow-up.

\section{DISCUSSION}

Tuberculosis (TB) is a worldwide spread infectious disease that is caused by Mycobacterium tuberculosis. Migrations, increased use of immunosuppressive agents and the epidemic of AIDS have contributed to its resurgence [3]. The most common form of presentation of $\mathrm{TB}$ is the pulmonary one. Lungs can be involved by bronchogenic or hematogenic dissemination. Abdominal involvement includes the intestine, peritoneum, and lymph nodes as the most common locations [4].

TB during pregnancy has been a concern since the days of Hypocrites. The incidence of TB in pregnancy is low [5]. Sometimes, the delayed diagnosis in pregnant women is due to vague symptoms that can be secondary to other diseases or even the growing of the fetus, a tendency to defer the use of radiological diagnostic tools, and difficulties in surgical approaches if needed. Presentation of TB in pregnant and non-pregnant women is similar, been pulmonary TB the most common presentation from [6].

A possible link between pregnancy and tuberculosis could be that pregnancy suppresses the T-helper (Th) 1 proinflammatory response. This suppression could both mask symptoms and increase susceptibility to new infection and reactivation of tuberculosis. Th1 suppression reverses after delivery, and symptoms could exacerbate [7].

Presentation of TB involving the abdominal cavity, also called abdominal tuberculosis (ATB) is uncommon. It is commonly found in patients who have severe and disseminated disease such as those resulting from miliary TB. This condition use to be secondary to lymphohematogenous spread from an affected lung, or direct invasion from an adjacent affected organ [7], [8]. In our patient, retroperitoneal location of TB was no associated to lung or spine involvement.

TB frequently affects non-immunocompetent hosts, especially HIV-infected and those with diabetes mellitus or end-stage renal disease [9]. In the case reported, immunosuppression could be excluded given the medical records, normal immunoglobulin levels and negative HIV test. Our living area is considered endemic. In this sense, reactivation of latent infection is possible. Delayed presentation and the formation of a large abscess, like in the present case, occur frequently [10].

ATB is a diagnostic challenge. This is especially difficult in the absence of associated lung involvement. ATB can mimic other diseases with abdominal involvement. In this sense, clinical presentation of ATB is usually non-specific resulting in diagnostic delay and the possible development of associated complications [4], [6], [11].

TB may be detected by using radiological test such as chest X-ray or computed tomography but there are no pathognomonic criteria [12], [13]. Polymerase chain reaction for Mycobacterium tuberculosis is a rapid and 
reliable test with relatively high sensibility $(76 \%)$ and much higher specificity $(99 \%)$. This is true for sputum samples, but tissue and other body fluid specimens have not been validated to date as reliable in large case series [12], [14], [15]. Diagnosis often requires a surgical approach to obtain tissue samples for microbiological and histological investigation [16], [17]. The study of specimen's biopsy revealed granulomas and giant cells with necrotic tissue.

Bacteriologic examination should always be performed. Positive culture can be found when histological examination is negative. Microbiological examination includes identification of acid-fast bacilli (Ziehl-Neelsen staining positive), positive culture for Mycobacterium tuberculosis and positive PCR for Mycobacterium tuberculosis complex [16]. Currently recommended treatment for ATB is conventional anti-TB therapy lasting a minimum of 6 months [4], [16].

\section{CONCLUSION}

Immuno-suppression related to pregnancy can lead to the development of infectious disease being clinical and radiological presentation similar to the one of highly immunocompromised patients. On the other hand, tubercular etiology of an abdominal mass should be kept in mind in immunocompetent host if coming from endemic areas. Improvement of an earlier diagnosis and treatment of peritoneal tuberculosis in pregnant women are important issues in terms of minimizing adverse obstetrical and neonatal adverse effects.

\section{REFERENCES}

[1] Chaudhari A, Desai P, Vadel M, Kaptan K. Evaluation of primary retroperitoneal masses by computed tomography scan. Int J Med Sci Public Health. 2016; 5:1423-1429.

[2] Scali EP, Chandler TM, Heffernan EJ, Coyle J, Harris AC, Chang SD. Primary retroperitoneal masses: what is the differential diagnosis? Abdom Imaging. 2015; 40:1887-1903.

[3] Kassu A, Mengistu G, Ayele B, Diro E, Mekonnen F, Ketema D, et al. Coinfection and clinical manifestations of tuberculosis in human immunodeficiency virus-infected and -uninfected adults at a teaching hospital, northwest Ethiopia. J Microbiol Immunol Infect. 2007; 40:116-122.

[4] Lazarus A, Thilagar B. Abdominal tuberculosis. Dis Mon. 2007; 53:32-38.

[5] Lee G, Kim S, Park I, Shin J, Kim S. Tuberculous peritonitis in pregnancy. J Obstet Gynaecol Res. 2005; 31:436-438.

[6] Mnyani C, McIntyre J. Tuberculosis in pregnancy. Bjog Int J Obstet Gynaecol. 2011; 118:226-231.

[7] Pasqualini L, Leli C, De Socio G, Mazzolla R, Cavaliere A, Schillaci $\mathrm{G}$, et al. Retroperitoneal abscess: an uncommon localization of tubercular infection. Infez Med. 2008; 16:230-232.

[8] Nissapatorn V, Kuppusamy I, Josephine F, Jamaiah I, Rohela M, Anuar A. Tuberculosis: a resurgent disease in immunosuppressed patients. Southeast Asian J Trop Med Public Health. 2006; 37 Suppl 3:153-160.

[9] Sonnenberg P, Glynn J, Fielding K, Murray J, Godfrey-Faussett P, Shearer S. How soon after infection with HIV does the risk of tuberculosis start to increase? A retrospective cohort study in South African gold miners. J Infect Dis. 2005; 191:150-158.

[10] Karoui S, Bibani N, Ouaz A, Serghini M, Chebbi F, Nouira K, et al. Retroperitoneal abscess: a rare localization of tubercular infection. Gastroenterol Res Pr. 2010; 2010:475130.

[11] Mathad J, Gupta A. Tuberculosis in pregnant and postpartum women: epidemiology, management, and research gaps. Clin Infect Dis Off Publ Infect Dis Soc Am. 2012; 55:1532-1549.
[12] Uzunkoy A, Harma M, Harma M. Diagnosis of abdominal tuberculosis: experience from 11 cases and review of the literature. World J Gastroenterol. 2004; 10:3647-3649.

[13] Hassan I, Brilakis ES, Thompson R, Que F. Surgical management of abdominal tuberculosis. J Gastrointest Surg Off J Soc Surg Aliment Tract. 2002; 6:862-867.

[14] Shah N, Moodley P, Babaria P, Moodley S, Ramtahal M, Richardson $\mathrm{J}$, et al. Rapid diagnosis of tuberculosis and multidrug resistance by the microscopic-observation drug-susceptibility assay. Am J Respir Crit Care Med. 2011; 183:1427-1433.

[15] Yun Y, Lee K, Haihua L, Ryu Y, Kim B, Lee Y, et al. Detection and identification of Mycobacterium tuberculosis in joint biopsy specimens by rpoB PCR cloning and sequencing. J Clin Microbiol. 2005; 43:174-178.

[16] Mamo J, Brij S, Enoch D. Abdominal tuberculosis: a retrospective review of cases presenting to a UK district hospital. Qjm Mon J Assoc Physicians. 2013; 106:347-354.

[17] Rai S, Thomas WM. Diagnosis of abdominal tuberculosis: the importance of laparoscopy. J R Soc Med. 2003; 96:586-588. 